\title{
Precooling, Planting Depth, and Shade Affect Cut Flower Quality and Perennialization of Field-grown Spring Bulbs
}

\author{
Todd J. Cavins ${ }^{1}$ and John M. Dole ${ }^{2}$ \\ Department of Horticulture and Landscape Architecture, Oklahoma State \\ University, Stillwater, OK 74078-6027
}

Additional index words. specialty cut flowers, Narcissus 'Music Hall', Narcissus 'Tahiti', Tulipa 'Couleur Cardinal', Tulipa 'White Emperor'

\begin{abstract}
Narcissus L. 'Music Hall', N. 'Tahiti', Tulipa L. 'Couleur Cardinal', and $T$. 'White Emperor' bulbs were precooled at $5{ }^{\circ} \mathrm{C}$ for 0 or 5 weeks and planted 15, 30, or $45 \mathrm{~cm}$ deep (from bulb base) into raised ground beds under $0 \%, 30 \%$, or $60 \%$ shade. Plant growth was monitored for two consecutive years after planting. Precooling reduced the percentage of $T$. 'White Emperor' that flowered but did not affect flowering percentage of the other cultivars. Precooling delayed anthesis in one or both years for all cultivars. The greatest percentage of bulbs flowered when planted $15 \mathrm{~cm}$ deep and the 45-cm planting depth reduced flowering percentage. Increasing planting depth delayed anthesis for all cultivars. Increasing shade increased stem lengths in one or both years for all cultivars, but did not influence flowering percentage. Perennialization was low for all cultivars regardless of treatment. Cultivar differences in perennialization occurred; in year 2 up to $30 \%$ of $N$. 'Tahiti' bulbs flowered vs. 32\% for 'Music Hall' and up to 30\% of $T$. 'White Emperor' bulbs flowered vs. only $22 \%$ of 'Couleur Cardinal'.
\end{abstract}

Numerous bulb species can be grown in southern Great Plains states, but are often used as annuals due to inconsistent perennialization. High bulb cost limits profitable cut flower production or annual landscape use. Bulb perennialization and cut flower harvests for two or more years would allow cost effective cut flower production. Factors affecting perennialization include inadequate duration of cold, improper planting depth, and excessive spring heating (Armitage, 1993; A. De Hertogh, personal communication).

Proper temperatures are the most important factor for bulb flowering and production (Buschman and Roozen, 1980; De Hertogh, 1996; Hartsema, 1961; Rees, 1972a). Precooling promotes rapid growth after planting and earlier shoot elongation (Le Nard and De Hertogh, 1993). Precooling also improves root development at temperatures as high as $30{ }^{\circ} \mathrm{C}$ compared to the optimum rooting temperature of 13 to $17{ }^{\circ} \mathrm{C}$ (Jennings and De Hertogh, 1977; Le Nard and De Hertogh, 1993).

Received for publication 24 Jan. 2000. Accepted for publication 20 July 2001. Approved for publication by the Director, Oklahoma Agricultural Experiment Station. Research supported in part by Stuifbergen Bloembollen Export B.V. and OAES under project H-2119. We thank Leah Aufill for her technical assistance.

${ }^{1}$ Graduate Research Assistant, currently at Dept. of Horticultural Science, North Carolina State Univ., Box 7609, Raleigh, NC 27695-7609.

${ }^{2}$ Professor, currently at Dept. of Horticultural Science, North Carolina State Univ., Box 7609, Raleigh, NC 27695-7609.
Daughter bulb formation and growth is essential for perennialization. Extended foliage life contributes to the photosynthate storage of bulbs. Aung et al. (1973) reported that $83 \%$ of the starch in the matured Tulipa bulblets was derived from photosynthesis. Early senescence of roots due to warm temperatures deprive bulbs of essential nutrients for development (Le Nard and De Hertogh, 1993). Plants that senesce rapidly during early hot dry summers have reduced bulb yields

Shallow planting causes poor rooting and increased probability of disease due to the higher soil temperatures (Le Nard and De Hertogh, 1993). Thus, deep planting depths may improve perennialization. Soil temperatures remain cool for long periods at deep depths. However, planting bulbs too deep delays emergence and flowering, and decreases bulb yield (Allen, 1938; Le Nard and De Hertogh, 1993; Wallis, 1964).

Finally, reducing light intensity by shading may reduce soil temperatures and protect the foliage from excessive heat. Shading also increases stem length (Armitage, 1991; Armitage et al., 1990; Gude et al., n.d.). Many plant species produce fewer flowers, but longer stems when grown under shade. However, when Anemone coronaria L. was grown under $67 \%$ shade, flower number per plant did not decrease and stem lengths were longer compared to $55 \%$ shade or ambient light levels (Armitage, 1991).

Narcissus and Tulipa have similar annual growing requirements and exhibit potential as field cut flowers. Narcissus are popular garden plants and Tulipa have long been the (Rees, 1972b). most popular of the bulbous species for cut flowers. Limited published information exists on the field production of Narcissus and Tulipa in Oklahoma or other southern Great Plains states; however, Narcissus generally naturalize well in Oklahoma (P. Mitchell, personal communication).

The objective of this study was to determine the effect of precooling, planting depth, and shade on cut flower characteristics and perennialization of Narcissus and Tulipa as cut flowers or landscape plants in the southern Great Plains region of the United States.

\section{Material and Methods}

Narcissus 'Music Hall' and 'Tahiti' and $T$. 'Couleur Cardinal' and 'White Emperor' bulbs were divided into two groups per cultivar. One group received 5 weeks of precooling at $5 \pm 1.5^{\circ} \mathrm{C}$ and the other group received no precooling. The precooling began at the same time, 19 Dec. 1997, that the nonprecooled bulbs were planted in the ground. Bulbs were stored at $18 \pm 1.0{ }^{\circ} \mathrm{C}$ prior to planting or precooling.

Bulbs were planted 15,30 , or $45 \mathrm{~cm}$ deep, measured from the soil surface to the bulb base in raised field beds of Norge Loam (finesilty, mixed, thermic Udic Paleustols) in Stillwater, Okla. [U.S. Dept. of Agriculture (USDA) climatic zone 6b-7a]. The soil was amended based on soil test results (Oklahoma State Univ. Soil, Water and Forage Testing Lab, Stillwater, Okla.) with $4 \mathrm{~g} \cdot \mathrm{m}^{-2}$ $\mathrm{NH}_{4} \mathrm{NO}_{3}$ for $\mathrm{N}, 25 \mathrm{~g} \cdot \mathrm{m}^{-2} \mathrm{CaCO}_{3}$ to adjust soil $\mathrm{pH}$ and $74 \mathrm{~g} \cdot \mathrm{m}^{-2}$ bone meal as a $\mathrm{P}$ source. Three levels of shade $(0 \%, 30 \%$, or $60 \%)$ provided average light intensities of 1480 , 831 , or $331 \mu \mathrm{mol} \cdot \mathrm{m}^{-2} \cdot \mathrm{s}^{-1}$, respectively. The woven polypropylene shade cloth covered the top ( $1 \mathrm{~m}$ above the soil surface) and south side of the raised beds.

Date of anthesis, stem length of flowering plants (cut and measured from the soil surface to the uppermost flower tip of Narcissus and from the node of the lowermost leaf, retained intact for photosynthesis, to the uppermost flower tip of Tulipa) at harvest, days to foliage senescence, and daily soil temperatures at 15, 30, and $45 \mathrm{~cm}$ deep from $19 \mathrm{Dec}$. 1997 to 20 July 1999 (Fig. 1) were collected. Cut flower quality was determined for each flowering stem on a scale of 1 to 5 with $1=\mathrm{a}$ thin, crooked stem with small flowers or incomplete flower opening and 5 = a straight, sturdy stem with large flower diameter.

The experiment was a split plot with shade treatment as the main plot and planting depth and cold pretreatment as the subplots. There were four replications with six bulbs/replication for $N$. 'Music Hall', eight bulbs/replication for $N$. 'Tahiti' and 11 bulbs/ replication for $T$. 'Couleur Cardinal' and 'White Emperor'. Data were collected for two consecutive growing seasons and analyzed by general linear model procedures with trend analysis for means separation of planting depth and shade (SAS Institute, Cary, N.C.). 


\section{Results}

Narcissus 'Music Hall' . Precooling did not affect flowering percentage (Table 1). However, precooling delayed anthesis in year 1 . Stem length, cut flower quality, and days to foliage senescence were not affected by precooling. Precooling had no effect in year 2 (data not presented). No interactions among precooling, shade, and planting depth occurred in year 1 or 2 .

Shading delayed anthesis in year 1 (Table 1). However, stem length and cut flower quality increased when bulbs were grown under shade in both years. Shading did not affect flowering percentage or foliage senescence.

Increasing planting depths linearly decreased flowering percentage and delayed anthesis in both years (Table 1). In year 1, quality and days to foliage senescence were not affected by planting depth. However, in year 2, deep planting depths reduced cut flower quality and promoted early foliage senescence. Planting depth did not affect stem length in either year.

Narcissus 'Tahiti'. Deep planting reduced the percentage of flowering in both years and in year 1 precooling also reduced flowering percentage, except for bulbs planted $45-\mathrm{cm}$ deep where precooling increased the percentage of flowering (Table 2). Deep planting delayed anthesis in both years. Precooling delayed anthesis except for bulbs planted 45 $\mathrm{cm}$ deep in year 1 where precooling decreased days to anthesis. In year 1, precooling decreased stem length, especially when bulbs were planted deep, while in year 2 shade increased stem length. In year 1, planting precooled bulbs deep decreased cut flower quality. In year 2, shading increased cut flower quality. In year 1, foliage senescence was not affected by treatments. However, in year 2 precooled bulbs grown under no shade and planted 15 or $30 \mathrm{~cm}$ deep senesced the earliest.

Tulipa 'Couleur Cardinal'. In year 1, precooling and deep planting depths reduced flowering percentage except for bulbs planted $45 \mathrm{~cm}$ deep where precooling increased flowering percentage (Table 3 ). In year 2 , deep planting depths again reduced the percentage of flowering, thus reduced perennialization. Precooling and deep planting depths delayed anthesis in both years, while in year 1 only, shading also delayed anthesis. In year 1, stems were shortest for precooled bulbs not grown under shade and planted $15 \mathrm{~cm}$ deep. In year 2, stems were shortest when not grown under shade and planted $15 \mathrm{~cm}$ deep. In year 1 , cut flower quality was highest when nonprecooled bulbs were planted $30 \mathrm{~cm}$ deep. In year 2, shade and deep planting increased quality ratings. In year 1, precooling and shade delayed foliage senescence, but no effect was evident in year 2.

Tulipa 'White Emperor'. In both years, precooling and $45-\mathrm{cm}$ planting depths reduced the percentage of flowering (Table 4). Increasing planting depth and precooling increased days to anthesis in both years. In year 2, shade delayed anthesis, but only for
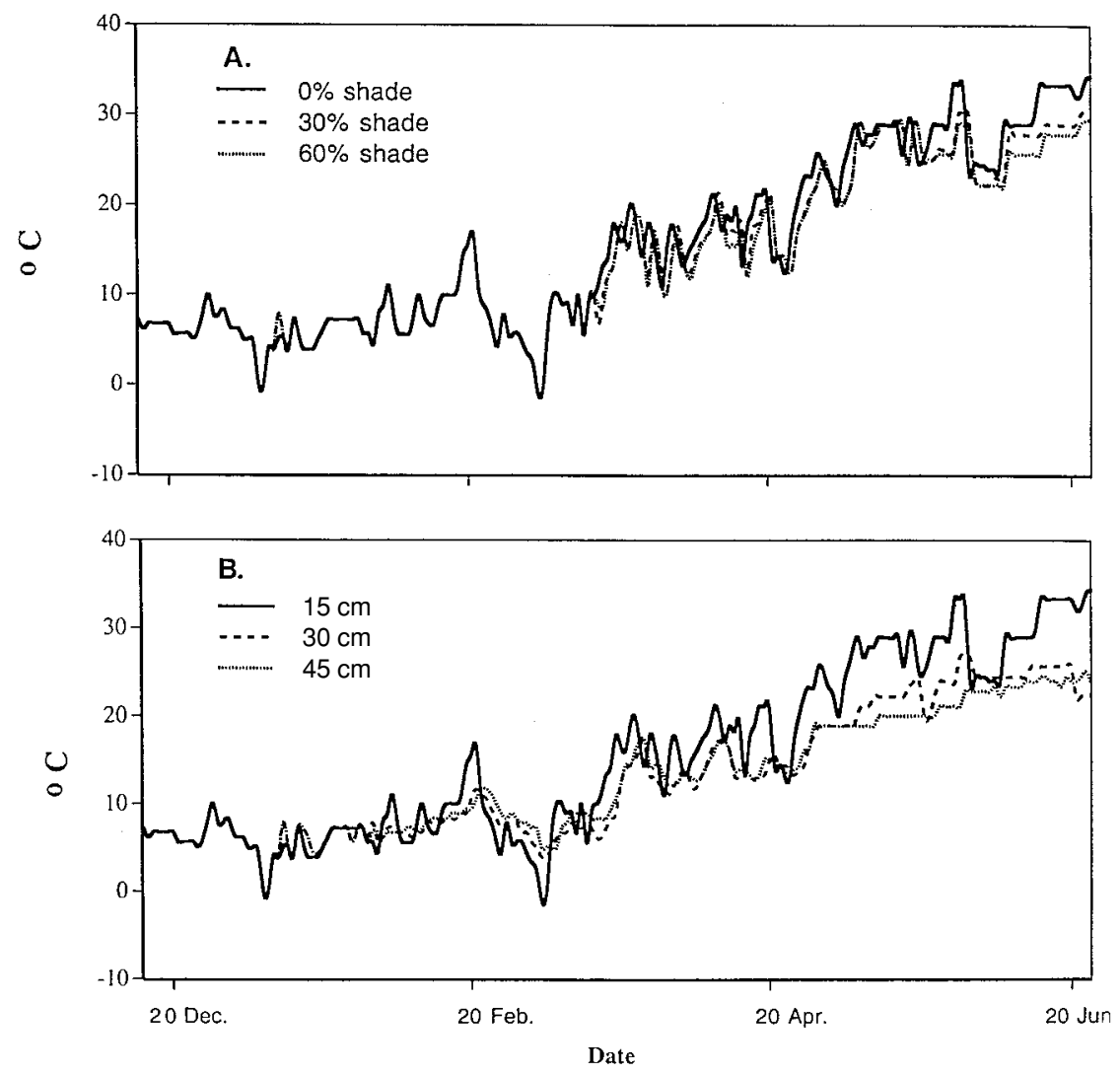

Fig. 1. (A) Soil temperatures $15 \mathrm{~cm}$ below surface with $0 \%, 30 \%$, or $60 \%$ shade; and (B) soil temperatures at 15,30 , and $45 \mathrm{~cm}$ below soil surface with no shade from Dec. 1997 to June 1998. Temperatures were significantly different among depths at $P<0.05$.

Table 1. Effect of precooling ${ }^{z}$, shade, and planting depth on cut flower characteristics and perennialization of Narcissus 'Music Hall'. There were 24 bulbs per treatment.

\begin{tabular}{|c|c|c|c|c|c|}
\hline Treatment & $\begin{array}{c}\text { Bulbs flowering } \\
(\%)\end{array}$ & $\begin{array}{l}\text { Days to } \\
\text { anthesis }^{y}\end{array}$ & $\begin{array}{l}\text { Stem length } \\
(\mathrm{cm})^{\mathrm{x}}\end{array}$ & $\begin{array}{l}\text { Quality } \\
\text { ratingw }\end{array}$ & $\begin{array}{c}\text { Days to foliage } \\
\text { senescence }^{y}\end{array}$ \\
\hline \multicolumn{6}{|c|}{ Year 1-Precooling } \\
\hline Precooled & 83 & 54 & 29.2 & 2.9 & 126 \\
\hline Nonprecooled & 67 & 51 & 29.5 & 3.0 & 126 \\
\hline Significance & NS & $* *$ & NS & NS & NS \\
\hline \multicolumn{6}{|c|}{ Year 1-Shade } \\
\hline $0 \%$ & 79 & 52 & 28.4 & 2.8 & 125 \\
\hline $30 \%$ & 82 & 52 & 29.8 & 3.0 & 125 \\
\hline $60 \%$ & 64 & 54 & 29.9 & 3.1 & 128 \\
\hline Significance & NS & $\mathrm{L}^{*}$ & $\mathrm{~L}^{* *}$ & $\mathrm{~L}^{*}$ & NS \\
\hline \multicolumn{6}{|c|}{ Year 1-Planting depth $(\mathrm{cm})$} \\
\hline 15 & 98 & 49 & 29.7 & 3.0 & 126 \\
\hline 30 & 84 & 54 & 29.8 & 3.0 & 125 \\
\hline 45 & 43 & 58 & 27.4 & 2.7 & 125 \\
\hline Significance & $\mathrm{L}^{* * *}$ & $\mathrm{~L}^{* * *}$ & NS & NS & NS \\
\hline \multicolumn{6}{|c|}{ Year 2-Shade } \\
\hline $0 \%$ & 14 & 21 & 30.2 & 3.3 & 144 \\
\hline $30 \%$ & 21 & 22 & 30.6 & 3.3 & 144 \\
\hline $60 \%$ & 19 & 20 & 32.3 & 3.7 & 143 \\
\hline Significance & NS & NS & $\mathrm{L}^{* * *}$ & $\mathrm{~L}^{* *}$ & NS \\
\hline \multicolumn{6}{|c|}{ Year $2-$ Planting depth $(\mathrm{cm})$} \\
\hline 15 & 32 & 19 & 31.2 & 3.5 & 144 \\
\hline 30 & 19 & 23 & 31.0 & 3.4 & 144 \\
\hline 45 & 3 & 26 & 30.6 & 2.9 & 143 \\
\hline Significance & $\mathrm{L}^{* *}$ & $\mathrm{~L}^{* * *}$ & NS & $\mathrm{L}^{*} \mathrm{Q}^{*}$ & $\mathrm{~L}^{*}$ \\
\hline
\end{tabular}

${ }^{2}$ Bulbs received 0 or 5 weeks of $5{ }^{\circ} \mathrm{C}$ precooling.

y Days from 20 Feb. 1998 in year 1 and 20 Feb. 1999 in year 2.

${ }^{x}$ Stem length was measured from the soil line to uppermost tip of the flower.

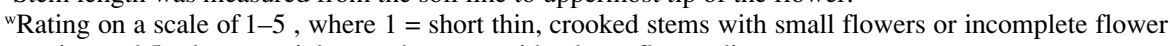
opening and $5=$ long, straight, sturdy stems with a large flower diameter.

ns, ${ }^{*}, * * * * * *$ Nonsignificant or significant at $P \leq 0.05,0.01,0.001$; linear (L), quadratic (Q). 
Table 2. Effect of precooling, shade, and planting depth on cut flower characteristics and perennialization of Narcissus 'Tahiti'. There were 32 bulbs per treatment.

\begin{tabular}{|c|c|c|c|c|c|c|c|}
\hline Precoooling ${ }^{\mathrm{z}}$ & $\begin{array}{l}\text { Shade } \\
(\%)\end{array}$ & $\begin{array}{c}\text { Planting } \\
\text { depth } \\
(\mathrm{cm})\end{array}$ & $\begin{array}{c}\text { Bulbs } \\
\text { flowering } \\
(\%)\end{array}$ & $\begin{array}{l}\text { Days to } \\
\text { anthesis }^{y}\end{array}$ & $\begin{array}{c}\text { Stem } \\
\text { length } \\
(\mathrm{cm})^{x}\end{array}$ & $\begin{array}{l}\text { Quality } \\
\text { rating }\end{array}$ & $\begin{array}{c}\text { Days to } \\
\text { foliage } \\
\text { senescence }^{y}\end{array}$ \\
\hline \multicolumn{8}{|c|}{ Year 1} \\
\hline \multirow[t]{9}{*}{ No } & 0 & 15 & 97 & 50 & 38.1 & 4.0 & 128 \\
\hline & & 30 & 94 & 53 & 33.3 & 3.4 & 128 \\
\hline & & 45 & 13 & 68 & 33.5 & 3.4 & 127 \\
\hline & 30 & 15 & 100 & 52 & 39.3 & 4.2 & 128 \\
\hline & & 30 & 91 & 56 & 32.4 & 3.1 & 128 \\
\hline & & 45 & 6 & 65 & 35.5 & 3.8 & 127 \\
\hline & 60 & 15 & 88 & 52 & 37.2 & 3.8 & 130 \\
\hline & & 30 & 84 & 55 & 33.6 & 3.1 & 128 \\
\hline & & 45 & 9 & 73 & 43.3 & 4.5 & 127 \\
\hline \multirow[t]{9}{*}{ Yes } & 0 & 15 & 84 & 53 & 32.3 & 3.4 & 127 \\
\hline & & 30 & 84 & 55 & 27.1 & 2.6 & 128 \\
\hline & & 45 & 44 & 60 & 26.1 & 2.4 & 126 \\
\hline & 30 & 15 & 97 & 53 & 32.3 & 3.3 & 125 \\
\hline & & 30 & 88 & 57 & 31.2 & 3.2 & 126 \\
\hline & & 45 & 28 & 62 & 27.7 & 2.6 & 128 \\
\hline & 60 & 15 & 94 & 54 & 31.3 & 3.1 & 128 \\
\hline & & 30 & 78 & 58 & 30.6 & 3.1 & 128 \\
\hline & & 45 & 31 & 67 & 27.6 & 2.9 & 128 \\
\hline \multicolumn{3}{|l|}{ Precooling $(\mathrm{P})$} & NS & $* * *$ & $* * *$ & $* * *$ & NS \\
\hline \multicolumn{3}{|l|}{ Shade $(\mathrm{S})$} & NS & $\mathrm{L}^{* *}$ & NS & NS & NS \\
\hline \multicolumn{3}{|c|}{ Depth (D) } & $\mathrm{L}^{* * * *}$ & $\mathrm{~L}^{* * *} \mathrm{Q}^{* * *}$ & $\mathrm{~L}^{* * * *}$ & $\mathrm{~L}^{* * *}$ & NS \\
\hline \multicolumn{3}{|c|}{ Significant $(P \leq 0.05)$ interactions } & $\mathrm{P} \times \mathrm{DL}$ & $\mathrm{P} \times \mathrm{DQ}$ & $\mathrm{P} \times \mathrm{DQ}$ & $\begin{array}{c}\mathrm{SL} \times \mathrm{DL} \\
\mathrm{P} \times \mathrm{DQ}\end{array}$ & None \\
\hline \multicolumn{8}{|c|}{ Year 2} \\
\hline \multirow[t]{9}{*}{ No } & 0 & 15 & 22 & 38 & 34.5 & 3.7 & 118 \\
\hline & & 30 & 21 & 41 & 35.5 & 3.6 & 124 \\
\hline & & 45 & 0 & --- & --- & --- & --- \\
\hline & 30 & 15 & 20 & 39 & 40.2 & 4.0 & 113 \\
\hline & & 30 & 22 & 41 & 38.2 & 3.8 & 124 \\
\hline & & 45 & 1 & 43 & 41.0 & 4.0 & 109 \\
\hline & 60 & 15 & 24 & 38 & 40.8 & 4.1 & 125 \\
\hline & & 30 & 22 & 42 & 40.8 & 4.0 & 126 \\
\hline & & 45 & 0 & --- & --- & --- & --- \\
\hline \multirow[t]{9}{*}{ Yes } & 0 & 15 & 22 & 43 & 34.4 & 3.8 & 113 \\
\hline & & 30 & 16 & 43 & 35.6 & 3.8 & 119 \\
\hline & & 45 & 6 & 44 & 30.0 & 3.1 & 124 \\
\hline & 30 & 15 & 30 & 42 & 38.2 & 4.0 & 112 \\
\hline & & 30 & 20 & 43 & 38.2 & 3.8 & 122 \\
\hline & & 45 & 10 & 45 & 33.9 & 3.5 & 128 \\
\hline & 60 & 15 & 24 & 42 & 39.1 & 4.0 & 120 \\
\hline & & 30 & 20 & 42 & 37.7 & 3.7 & 123 \\
\hline & & 45 & 4 & 45 & 37.5 & 4.0 & 128 \\
\hline \multicolumn{3}{|l|}{ Precooling $(\mathrm{P})$} & NS & $* * *$ & NS & NS & NS \\
\hline \multicolumn{3}{|l|}{ Shade $(\mathrm{S})$} & NS & NS & $\mathrm{L}^{* * * *} \mathrm{Q}^{*}$ & $\mathrm{~L}^{*}$ & NS \\
\hline \multicolumn{3}{|c|}{ Depth (D) } & $\mathrm{L}^{* * * *}$ & $\mathrm{~L}^{* * * *}$ & NS & $\mathrm{L}^{*}$ & $\mathrm{~L}^{*}$ \\
\hline \multicolumn{3}{|c|}{ Significant $(P \leq 0.05)$ interactions } & None & $\mathrm{P} \times \mathrm{DL}$ & None & None & $\begin{array}{c}\mathrm{P} \times \mathrm{DQ} \\
\mathrm{P} \times \mathrm{SL} \times \mathrm{DL}\end{array}$ \\
\hline
\end{tabular}

${ }^{2}$ Bulbs received 0 or 5 weeks of $5{ }^{\circ} \mathrm{C}$ precooling.

${ }^{y}$ Days from 20 Feb. 1998 in year 1 and 20 Feb. 1999 in year 2.

x Stem length was measured from the soil line to uppermost tip of the flower.

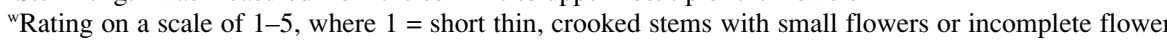
opening and $5=$ long, straight, sturdy stems with a large flower diameter.

Ns, ${ }^{* * * * * * *}$ Nonsignificant or significant at $P \leq 0.05,0.01,0.001$; linear (L), quadratic (Q).

precooled bulbs. In year 1 , stem lengths decreased with increasing planting depth, except for nonprecooled bulbs grown without shade. In year 2, shading increased stem lengths. Quality ratings were lowest when bulbs were planted $45 \mathrm{~cm}$ deep, especially with nonprecooled bulbs. In year 2 , shade increased quality except for precooled bulbs planted $15 \mathrm{~cm}$ deep. In year 1 , foliage senescence was delayed when precooled bulbs were planted 30 or $45 \mathrm{~cm}$ deep and grown under shade. In year 2 , precooled bulbs planted $45 \mathrm{~cm}$ deep had the greatest number of days to foliage senescence.

\section{Discussion}

Precooling and deep planting reduced flowering percentage in year 1 for all cultivars and species except $N$. 'Music Hall' where only deep planting reduced flowering percentage. Increased planting depth decreased flowering percentage which was noted also by Fodor (1974), Van der Knaap (1969), Van Ouwerkerk (1969) and Wallis (1964). In contrast, $N$. 'Tahiti' and $T$. 'Couleur Cardinal' flowering percentage increased at $45-\mathrm{cm}$ planting depths with precooling. Bulbs planted at $45 \mathrm{~cm}$ produced few or no flowers. Thus, the increase in flower- ing percentage from precooling for $N$. 'Tahiti' and $T$. 'Couluer Cardinal' was not commercially useful. Bulbs planted at $15 \mathrm{~cm}$ generally produced more flowers than at $30 \mathrm{~cm}$.

Precooling and deep planting depths also delayed anthesis. Le Nard and De Hertogh (1993) noted that days to anthesis were generally reduced when bulbs were precooled. However, in our study anthesis was delayed by precooling in one or both years for all cultivars (Tables 1, 2, 3, and 4). Nonprecooled bulbs were planted five weeks prior to precooled bulbs. Earlier planting probably allowed the nonprecooled bulbs to produce roots while precooled bulbs were still in a cooler. These results were similar to those of Van Staden (1978) who reported Narcissus root well without precooling and Le Nard and Cohat (1968) who noted Tulipa grow well at optimum planting temperatures without precooling.

Bulbs planted 30 or $45 \mathrm{~cm}$ deep required more days to reach anthesis compared to the $15 \mathrm{~cm}$ planting depth for all cultivars (Tables $1,2,3$, and 4). This was also noted by Allen (1938), Le Nard and De Hertogh (1993), and Wallis (1964). Anthesis was also delayed in year 1 for $N$. 'Music Hall', $N$. 'Tahiti', and $T$. 'Couleur Cardinal' by shading which possibly slowed plant growth (Fig. 1).

For all cultivars except $T$. 'White Emperor', precooling did not affect perennialization. The daughter bulb was not present during precooling, so no affect would be expected. Precooling was significant for $T$. 'White Emperor' in year 2, but likely as a residual effect from year 1 .

Long stems were generally produced from nonprecooled bulbs grown under shade. However, Le Nard and De Hertogh (1993) noted that precooling generally produced longer stems. In our study, the nonprecooled bulbs may have received sufficient cooling from ground temperatures (Fig. 1). Shading reduced light intensity and increased stem length which has also been reported on Anemone coronaria L., Centaurea americana Nutt., Echinops ritro L., Eryngium planum L., Oxypetalum caeruleum (D. Don) Decne., Tulipa L. and Zantedeschia Spreng. (Armitage, 1991; Armitage et al., 1990; Gude et al., n.d.) Cut flower quality was increased by shading which reduced light intensities and may have reduced heat stress.

Bulbs planted 15 or $30 \mathrm{~cm}$ deep generally had longer stems than bulbs planted $45 \mathrm{~cm}$ deep (Table 3). These results are similar to those obtained for Narcissus by Wallis (1964). Occasionally, bulbs planted $45 \mathrm{~cm}$ deep had longer stems compared to bulbs planted 15 or $30 \mathrm{~cm}$ deep; however, in those instances flowering percentages were low (1\% to 9\%) which may have produced misleading results. Planting depth had no effect on $N$. 'Music Hall' stem length.

Foliage senescence was delayed for precooled Tulipa bulbs. Precooling may have improved root development and prolonged foliage life (Jennings and De Hertogh, 1977; Le Nard and De Hertogh, 1993).

Deep planting delayed foliage senescence for $N$. 'Music Hall' (year 2), N. 'Tahiti' (year 2), and $T$. 'White Emperor' (both years) by 
Table 3. Effect of precooling, shade, and planting depth on cut flower characteristics and perennialization of Tulipa 'Couleur Cardinal'. There were 44 bulbs per treatment.

\begin{tabular}{|c|c|c|c|c|c|c|c|}
\hline Precooling ${ }^{\mathrm{z}}$ & $\begin{array}{c}\text { Shade } \\
(\%)\end{array}$ & $\begin{array}{l}\text { Planting } \\
\text { depth } \\
(\mathrm{cm})\end{array}$ & $\begin{array}{c}\text { Bulbs } \\
\text { flowering } \\
(\%)\end{array}$ & $\begin{array}{l}\text { Days to } \\
\text { anthesis }^{y}\end{array}$ & $\begin{array}{l}\text { Stem } \\
\text { length } \\
(\mathrm{cm})^{x}\end{array}$ & $\begin{array}{l}\text { Quality } \\
\text { rating }\end{array}$ & $\begin{array}{c}\text { Days to } \\
\text { foliage } \\
\text { senescence }^{y}\end{array}$ \\
\hline \multicolumn{8}{|c|}{ Year 1} \\
\hline \multirow[t]{9}{*}{ No } & 0 & 15 & 82 & 44 & 13.8 & 1.6 & 95 \\
\hline & & 30 & 70 & 49 & 15.0 & 1.9 & 91 \\
\hline & & 45 & 0 & --- & --- & --- & --- \\
\hline & 30 & 15 & 84 & 46 & 15.4 & 1.6 & 98 \\
\hline & & 30 & 86 & 49 & 17.1 & 2.3 & 100 \\
\hline & & 45 & 0 & --- & --- & --- & --- \\
\hline & 60 & 15 & 89 & 45 & 16.4 & 1.9 & 98 \\
\hline & & 30 & 73 & 51 & 18.2 & 2.4 & 101 \\
\hline & & 45 & 9 & 59 & 12.3 & 1.5 & 99 \\
\hline \multirow[t]{9}{*}{ Yes } & 0 & 15 & 70 & 55 & 10.3 & 0.9 & 97 \\
\hline & & 30 & 56 & 56 & 15.0 & 1.6 & 99 \\
\hline & & 45 & 23 & 59 & 11.7 & 1.6 & 99 \\
\hline & 30 & 15 & 86 & 58 & 12.6 & 1.2 & 103 \\
\hline & & 30 & 77 & 60 & 12.0 & 1.2 & 106 \\
\hline & & 45 & 30 & 66 & 11.9 & 1.3 & 102 \\
\hline & 60 & 15 & 84 & 58 & 13.0 & 1.4 & 104 \\
\hline & & 30 & 50 & 63 & 11.1 & 1.0 & 105 \\
\hline & & 45 & 25 & 65 & 12.5 & 1.4 & 102 \\
\hline \multicolumn{3}{|c|}{ Precooling $(\mathrm{P})$} & NS & $* * *$ & $* * *$ & $* * *$ & $* * *$ \\
\hline \multicolumn{3}{|c|}{ Shade $(S)$} & NS & $\mathrm{L}^{*}$ & NS & NS & $\mathrm{L}^{*}$ \\
\hline \multicolumn{3}{|l|}{ Depth (D) } & $\mathrm{L}^{* * *}$ & $\mathrm{~L}^{* * *} \mathrm{Q}^{* * *}$ & $\mathrm{Q}^{* *}$ & $\mathrm{Q}^{* *}$ & NS \\
\hline \multicolumn{3}{|c|}{ Significant $(P \leq 0.05)$ interactions } & $\mathrm{P} \times \mathrm{DL}$ & $\begin{array}{l}\mathrm{SL} \times \mathrm{DL} \\
\mathrm{P} \times \mathrm{DL}\end{array}$ & $\begin{array}{c}\mathrm{P} \times \mathrm{SL} \\
\mathrm{P} \times \mathrm{SL} \times \mathrm{DL}\end{array}$ & $\mathrm{P} \times \mathrm{SL} \times \mathrm{DL}$ & None \\
\hline \multicolumn{8}{|c|}{ Year 2} \\
\hline \multirow[t]{9}{*}{ No } & 0 & 15 & 22 & 44 & 16.4 & 1.3 & 94 \\
\hline & & 30 & 7 & 46 & 19.8 & 2.1 & 94 \\
\hline & & 45 & 0 & --- & --- & --- & --- \\
\hline & 30 & 15 & 20 & 46 & 19.0 & 1.8 & 99 \\
\hline & & 30 & 6 & 49 & 22.6 & 2.6 & 104 \\
\hline & & 45 & 0 & --- & --- & --- & --- \\
\hline & 60 & 15 & 22 & 46 & 19.1 & 1.8 & 99 \\
\hline & & 30 & 3 & 49 & 24.0 & 2.8 & 96 \\
\hline & & 45 & 0 & --- & --- & --- & --- \\
\hline \multirow[t]{9}{*}{ Yes } & 0 & 15 & 5 & 47 & 17.8 & 1.5 & 99 \\
\hline & & 30 & 6 & 48 & 20.4 & 2.3 & 100 \\
\hline & & 45 & 3 & 48 & 21.8 & 2.4 & 102 \\
\hline & 30 & 15 & 5 & 47 & 22.8 & 2.7 & 97 \\
\hline & & 30 & 8 & 48 & 24.3 & 3.1 & 99 \\
\hline & & 45 & 0 & --- & --- & --- & --- \\
\hline & 60 & 15 & 6 & 48 & 21.9 & 2.6 & 97 \\
\hline & & 30 & 3 & 48 & 20.3 & 2.7 & 102 \\
\hline & & 45 & 2 & 50 & 26.0 & 3.3 & 83 \\
\hline \multicolumn{3}{|c|}{ Precooling $(\mathrm{P})$} & NS & $*$ & $* *$ & NS & NS \\
\hline \multicolumn{3}{|c|}{ Shade $(S)$} & NS & $\mathrm{L}^{* * *}$ & $\mathrm{~L}^{* * * *} \mathrm{Q}^{*}$ & $\mathrm{~L}^{* *}$ & NS \\
\hline \multicolumn{3}{|c|}{ Depth (D) } & $\mathrm{L}^{* * *}$ & $\mathrm{~L}^{* * *}$ & $\mathrm{~L}^{* *}$ & $\mathrm{~L}^{* *}$ & NS \\
\hline \multicolumn{3}{|c|}{ Significant $(P \leq 0.05)$ interactions } & None & $\mathrm{P} \times \mathrm{DL}$ & None & None & None \\
\hline
\end{tabular}

${ }^{2}$ Bulbs received 0 or 5 weeks of $5{ }^{\circ} \mathrm{C}$ precooling.

${ }^{y}$ Days from 20 Feb. 1998 in year 1 and 20 Feb. 1999 in year 2.

${ }^{\mathrm{x}}$ Stem length was measured from node of wrapper leaf to uppermost tip of the flower.

${ }^{\text {w }}$ Rating on a scale of $1-5$, where $1=$ short thin, crooked stems with small flowers or incomplete flower opening and $5=$ long, straight, sturdy stems with a large flower diameter.

Ns, ${ }^{* * * * * * * *}$ Nonsignificant or significant at $P \leq 0.05,0.01,0.001$; linear (L), quadratic (Q).

delaying the increase of soil temperatures and may have improved rooting which extended foliage life (Fig. 1) (Le Nard and De Hertogh, 1993).

Shading extended foliage life for $T$. 'White Emperor' and T. 'Couleur Cardinal' bulbs planted $15 \mathrm{~cm}$ deep in year 1 . The lower light level may have reduced foliage temperatures allowing it to live longer.

In conclusion, the $45-\mathrm{cm}$ planting depth reduced flowering. Increasing planting depth delayed anthesis; while shading increased stem length and delayed anthesis. For cut flower production, the $15-\mathrm{cm}$ planting depth was suf- ficient, $30 \%$ to $60 \%$ shade lengthened stems and precooling was unnecessary. To prolong the cut flower harvest period of a specific cultivar, bulbs can be planted both 15 and $30 \mathrm{~cm}$ deep. However, deep planting $(45 \mathrm{~cm})$ may reduce flowering percentage.

Precooling, deep planting, and shade were not sufficient to encourage perennialization. The highest percentage of plants flowering in year 2 occurred with bulbs planted $15 \mathrm{~cm}$ deep. Precooling influenced percentage of plants flowering for $T$. 'White Emperor' but did not influence any other cultivars and does not appear to be commercially useful to promote perennialization of spring flowering bulbs in the southern Great Plains region of the United States. Overall perennialization was low, regardless of treatment, as $32 \%$ or less of bulbs survived to the second year. Species and cultivar selection may be most important in perennialization as the highest percentage of bulbs flowering in the second year was $30 \%$ for N. 'Tahiti' vs. $32 \%$ for N. 'Music Hall' and 30\% for $T$. 'White Emperor' vs. $22 \%$ for $T$. 'Couleur Cardinal'. Interestingly of the four species studied, Narcissus were considered the best for perennialization in the southern Great Plains, yet Tulipa performed as well as Narcissus (Armitage, 1993; P. Mitchell, personal communication). Apparently Narcissus cultivars other than the ones used in this study perform well in the southern Great Plains.

\section{Literature Cited}

Allen, R.C. 1938. Factors affecting the growth of tulips and narcissi in relation to garden practice. Proc Amer. Soc. Hort. Sci. 35:825-829.

Armitage, A.M. 1991. Shade effects yield and stem length of field-grown cut-flower species. HortScience 26:1174-1176.

Armitage, A.M. 1993. Tulipa, p. 311-315. In: Specialty cut flowers. Varsity Press, Portland, Ore.

Armitage, A.M., N.G. Seager, I.J. Warrington, and D.H. Greer. 1990. Response of Oxypetalum caeruleum to irradiance, temperature and photoperiod. J. Amer. Soc. Hort. Sci. 115:910 914

Aung, L.H., F. Tognoni, and A.A. De Hertogh. 1973. Changes in the carbohydrates of tulip bulbs during development. HortScience 8:207-208.

Buschman, J.C.M. and F.M. Roozen. 1980. Introduction, p. 3. In: Forcing flowerbulbs. Intl. Flower Bulb Centre, Hillegom, The Netherlands.

De Hertogh, A.A. 1996. Allium giganteum, p. D1314. In: Holland bulb forcer's guide, 5th ed. Intl. Flower Bulb Center, Hillegom, The Netherlands.

Fodor, B. 1974. Bulb yield of tulip in connection with row-distance and planting depth. Kerteszeti Egyetem Kozlemenyei 38:241-247.

Gude, H., K. de Jong, and P. Vreeburg. n.d. The forcing of tulip and hyacinth under artificial light. Laboratory voor Bloembollenonderzoek, Bulb Research Center, Lisse, The Netherlands.

Hartsema, A.H. 1961. Influence of temperatures on flower formation and flowering of bulbous and tuberous plants. In: W. Ruhland (ed.). Hanbuch der pflanzenphysiologie. Springer-Verlag, Berlin 16:123-167.

Jennings, N.T. and A.A. De Hertogh. 1977. The influence of preplanting dips and postplanting temperatures on root growth and development of nonprecooled tulips, daffodils and hyacinths. Scientia Hort. 6:157-166.

Le Nard, M. and J. Cohat. 1968. Influence des temperatures de conservation des bulbes sur l'elongation, la floraison et la bulbification de la tulip (Tulipa gesneria L.). Anales de l'Amelioration des Plantes 18:181-215.

Le Nard, M. and A.A. De Hertogh. 1993. Tulipa, p. 617-682. In: The physiology of flower bulbs: A comprehensive treatise on the physiology and utilization of ornamental flowering bulbous and tuberous plants. Elsevier, N.Y.

Rees, A.R., 1972a. Flower initiation and differentiation, p. 98-115. In: J.F. Sutcliffe (ed.). The growth of bulbs, vol I. Academic, London.

Rees, A.R. 1972b. Structure morphology development and periodicity, p. 17-44. In: J.F. Sutcliffe 
Table 4. Effect of precooling, shade, and planting depth on cut flower characteristics and perennialization of Tulipa 'White Emperor'. There were 44 bulbs per treatment.

\begin{tabular}{|c|c|c|c|c|c|c|c|}
\hline$\underline{\text { Precooling }}^{\mathrm{z}}$ & $\begin{array}{c}\text { Shade } \\
(\%)\end{array}$ & $\begin{array}{c}\text { Planting } \\
\text { depth } \\
(\mathrm{cm})\end{array}$ & $\begin{array}{c}\text { Bulbs } \\
\text { flowering } \\
(\%) \\
\end{array}$ & $\begin{array}{l}\text { Days to } \\
\text { anthesis }^{y}\end{array}$ & $\begin{array}{c}\text { Stem } \\
\text { length } \\
(\mathrm{cm})^{\mathrm{x}}\end{array}$ & $\begin{array}{l}\text { Quality } \\
\text { ratingw }^{\mathrm{w}}\end{array}$ & $\begin{array}{c}\text { Days to } \\
\text { foliage } \\
\text { senescence }^{y}\end{array}$ \\
\hline \multicolumn{8}{|c|}{ Year 1} \\
\hline \multirow[t]{9}{*}{ No } & 0 & 15 & 82 & 43 & 20.8 & 2.7 & 93 \\
\hline & & 30 & 84 & 48 & 21.2 & 2.5 & 96 \\
\hline & & 45 & 0 & --- & --- & --- & --- \\
\hline & 30 & 15 & 80 & 43 & 23.4 & 2.7 & 96 \\
\hline & & 30 & 82 & 46 & 22.2 & 2.6 & 96 \\
\hline & & 45 & 9 & 55 & 16.5 & 1.6 & 94 \\
\hline & 60 & 15 & 82 & 43 & 23.2 & 2.8 & 98 \\
\hline & & 30 & 77 & 47 & 22.4 & 2.8 & 100 \\
\hline & & 45 & 2 & 56 & 10.0 & 1.0 & 94 \\
\hline \multirow[t]{9}{*}{ Yes } & 0 & 15 & 61 & 45 & 22.9 & 2.8 & 92 \\
\hline & & 30 & 52 & 49 & 21.3 & 2.4 & 90 \\
\hline & & 45 & 16 & 52 & 16.7 & 1.9 & 90 \\
\hline & 30 & 15 & 59 & 47 & 20.5 & 2.4 & 100 \\
\hline & & 30 & 57 & 49 & 21.3 & 2.5 & 100 \\
\hline & & 45 & 18 & 54 & 16.3 & 1.9 & 100 \\
\hline & 60 & 15 & 64 & 51 & 19.4 & 2.1 & 96 \\
\hline & & 30 & 48 & 49 & 23.4 & 2.8 & 102 \\
\hline & & 45 & 34 & 53 & 19.1 & 2.2 & 104 \\
\hline \multicolumn{3}{|c|}{ Precooling $(\mathrm{P})$} & $*$ & $* * *$ & NS & NS & NS \\
\hline \multicolumn{3}{|c|}{ Shade $(\mathrm{S})$} & NS & NS & NS & NS & $\mathrm{L}^{* *}$ \\
\hline \multicolumn{3}{|c|}{ Depth (D) } & $\mathrm{L}^{* * *}$ & $\mathrm{~L}^{* * *} \mathrm{Q}^{*}$ & $\mathrm{~L}^{*} \mathrm{Q}^{* *}$ & $\mathrm{~L}^{*} \mathrm{Q}^{* *}$ & NS \\
\hline \multirow{2}{*}{\multicolumn{3}{|c|}{ Significant $(P \leq 0.05)$ interactions }} & $\mathrm{P}^{*} \mathrm{DL}$ & $\mathrm{P} \times \mathrm{SL}$ & $\mathrm{P} \times \mathrm{SL}$ & $\mathrm{P} \times \mathrm{DL}$ & $\mathrm{SL} \times \mathrm{DL}$ \\
\hline & & & & $\begin{array}{l}\mathrm{P} \times \mathrm{DL} \\
\mathrm{P} \times \mathrm{DL}\end{array}$ & \multicolumn{3}{|c|}{$\mathrm{P} \times \mathrm{SL} \times \mathrm{DL}$} \\
\hline \multicolumn{8}{|c|}{ Year 2} \\
\hline \multirow[t]{9}{*}{ No } & 0 & 15 & 22 & 33 & 22.4 & 2.4 & 80 \\
\hline & & 30 & 12 & 34 & 20.3 & 1.9 & 81 \\
\hline & & 45 & 0 & --- & --- & --- & --- \\
\hline & 30 & 15 & 28 & 34 & 24.6 & 2.4 & 85 \\
\hline & & 30 & 21 & 37 & 24.1 & 2.5 & 83 \\
\hline & & 45 & 2 & 38 & 27.0 & 3.5 & 83 \\
\hline & 60 & 15 & 30 & 34 & 25.4 & 2.6 & 82 \\
\hline & & 30 & 20 & 37 & 25.2 & 3.0 & 83 \\
\hline & & 45 & 0 & --- & --- & --- & --- \\
\hline \multirow[t]{9}{*}{ Yes } & 0 & 15 & 15 & 36 & 23.4 & 2.8 & 82 \\
\hline & & 30 & 11 & 36 & 23.3 & 2.6 & 84 \\
\hline & & 45 & 0 & --- & --- & --- & --- \\
\hline & 30 & 15 & 14 & 37 & 23.1 & 2.7 & 83 \\
\hline & & 30 & 17 & 38 & 23.3 & 2.6 & 84 \\
\hline & & 45 & 0 & --- & --- & --- & --- \\
\hline & 60 & 15 & 17 & 36 & 24.7 & 2.7 & 84 \\
\hline & & 30 & 11 & 38 & 27.8 & 3.3 & 88 \\
\hline & & 45 & 3 & 39 & 24.0 & 3.0 & 90 \\
\hline \multicolumn{3}{|c|}{ Precooling $(\mathrm{P})$} & $* *$ & $* *$ & NS & NS & $*$ \\
\hline \multicolumn{3}{|c|}{ Shade $(\mathrm{S})$} & NS & $\mathrm{L}^{* *} \mathrm{Q}^{* *}$ & $\mathrm{~L}^{* * *}$ & $\mathrm{~L}^{*}$ & NS \\
\hline \multicolumn{3}{|c|}{ Depth (D) } & $\mathrm{L}^{* * * *}$ & $\mathrm{~L}^{* * *}$ & NS & NS & $\mathrm{L}^{* *}$ \\
\hline \multicolumn{3}{|c|}{ Significant $(P \leq 0.05)$ interactions } & None & $\begin{array}{c}\mathrm{SL} \times \mathrm{DL} \\
\mathrm{P} \times \mathrm{DL}\end{array}$ & None & $\mathrm{SL} \times \mathrm{DL}$ & None \\
\hline
\end{tabular}

${ }^{\mathrm{z}}$ Bulbs received 0 or 5 weeks of $5{ }^{\circ} \mathrm{C}$ precooling.

${ }^{y}$ Days from 20 Feb. 1998 in year 1 and 20 Feb. 1999 in year 2.

${ }^{x}$ Stem length was measured from the node of the wrapper leaf to uppermost tip of the flower.

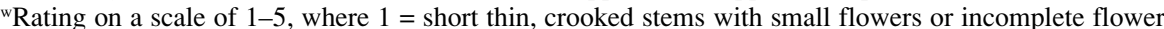
opening and $5=$ long, straight, sturdy stems with a large flower diameter.

ss, *, **, ${ }^{* * *}$ Nonsignificant or significant at $P \leq 0.05,0.01,0.001$; linear (L), quadratic (Q). (ed.). The growth of bulbs, vol I. Academic, London.

Van der Knaap, W. 1969. Opbrengstderving bij tulpen door planten op ongelijke en te grote depte. Weekblad voor Bloembollencultuur 80:367-368.

Van Ouwerkerk, C. 1969. Invloed van de relatieve boldiepte op de opbengst van tulpen. Tuinbouw Mededeelingen 32:398-403.

Van Staden, J. 1978. The levels of endogenous cytokinins in daffodil bulbs stored under different environmental conditions. Zeitschrift furPflansenphysiologie 86:323-330.

Wallis, L.W. 1964. Bulbs. Rosewarne Expt. Hort. Sta. Annu. Rpt. 1963:14-31. 